2016-10-01

\title{
Analysis of the elemental composition of marine litter by field-portable-XRF
}

\section{Turner, Andrew}

http://hdl.handle.net/10026.1/5367

10.1016/j.talanta.2016.06.026

Talanta

Elsevier BV

All content in PEARL is protected by copyright law. Author manuscripts are made available in accordance with publisher policies. Please cite only the published version using the details provided on the item record or document. In the absence of an open licence (e.g. Creative Commons), permissions for further reuse of content should be sought from the publisher or author. 
5

6

16

17 *Corresponding author. Tel: +44 1752 584570; Fax: +44 1752 584710; e-mail: 18 aturner@plymouth.ac.uk by field-portable-XRF

\author{
Andrew Turner* \& Kevin R. Solman \\ School of Geography, Earth and Environmental Sciences \\ Plymouth University \\ Drake Circus \\ Plymouth PLA 8AA \\ $U K$
}

19

20

21 


\section{Abstract}

Marine litter represents a pervasive environmental problem that poses direct threats to wildlife and habitats. Indirectly, litter can also act as a vehicle for the exposure and bioaccumulation of chemicals that are associated with manufactured or processed solids. In this study, we describe the use of a Niton field-portable-X-ray fluorescence (FP-XRF) spectrometer to determine the content of 17 elements in beached plastics, foams, ropes and painted items. The instrument was used in a 'plastics' mode configured for complex, low density materials, and employed a thickness correction algorithm to account for varying sample depth. Accuracy was evaluated by analysing two reference polyethylene discs and was better than $15 \%$ for all elements that had been artificially impregnated into the polymer. Regarding the litter samples, limits of detection for a 120 second counting time varied between the different material categories and among the elements but were generally lowest for plastics and painted items with median concentrations of less than $10 \mu \mathrm{g} \mathrm{g} \mathrm{g}^{-1}$ for $\mathrm{As}, \mathrm{Bi}, \mathrm{Br}, \mathrm{Cr}, \mathrm{Hg}, \mathrm{Ni}, \mathrm{Pb}$, Se and Zn. Concentrations returned by the XRF were highly sensitive to the thickness correction applied for certain elements $(\mathrm{Ba}, \mathrm{Cl}, \mathrm{Cr}, \mathrm{Cu}, \mathrm{Fe}, \mathrm{Sb}, \mathrm{Ti}, \mathrm{Zn})$ in all matrices tested, indicating that accurate measurement and application of the correct thickness is critical for acquiring reliable results. An independent measure of the elemental content of selected samples by ICP spectrometry following acid digestion returned concentrations that were significantly correlated with those returned by the XRF, and with an overall slope of $[\mathrm{XRF}] /[\mathrm{ICP}]=0.85$. Within the FP-XRF operating conditions, $\mathrm{Cl}, \mathrm{Cr}, \mathrm{Fe}$, Ti and $\mathrm{Zn}$ were detected in more than $50 \%$ and $\mathrm{Hg}$ and $\mathrm{Se}$ in less than $1 \%$ of the 367 litter samples analysed. Significant from an environmental perspective were concentrations of the hazardous elements, $\mathrm{Cd}, \mathrm{Br}$ and $\mathrm{Pb}$, that exceeded several thousand $\mu \mathrm{g} \mathrm{g} \mathrm{g}^{-1}$ in many cases. 
48 Keywords: marine litter; XRF; plastics; ropes; foams; metals 


\section{Introduction}

50 The accumulation of marine litter in the open ocean and on beaches is a significant

51 and pervasive global problem. Discarded solid waste has a multitude of both land-

52 based and marine-based sources and arises through a lack of awareness and poor or

53 ineffective waste management practices. As well as representing an aesthetic problem

54 on the foreshore and a nuisance to boaters, manufactured or processed items of waste

55 that are composed of persistent, durable and low-density materials, like plastics,

56 rubbers and ropes, pose serious threats to wildlife through ingestion, suffocation and

57 entanglement [1]. Ingestion of small fragments of litter also affords a route of

58 exposure to and accumulation of chemicals that are associated with these materials

59 [2]. Such chemicals are either an inherent component or degradation product of the

60 solid itself, or may have accumulated from sea water during suspension and

61 transportation of the litter [3].

Although hydrophobic organic pollutants, including polychlorinated biphenyls and polycyclic aromatic hydrocarbons, have been well-studied in this respect [4-6], recent attention has also focussed on the association of various trace metals, like $\mathrm{Cd}, \mathrm{Cu}, \mathrm{Cr}$, $\mathrm{Ni}, \mathrm{Pb}$ and $\mathrm{Zn}$, with plastic litter. Thus, controlled laboratory experiments have shown that small quantities of metal can rapidly adsorb onto the plastic surface from sea water [7] while mild acid digestion of beached plastics has revealed that greater quantities of metals can gradually accumulate in natural films coating the polymer [8]. More important from a bulk concentration perspective, however, is the association of

71 certain metals with plastics and other synthetic solids arising from the manufacturing 72 process itself [9]. For example, many metals and metalloids and their compounds are 73 used, or have been used before restrictions came into place, as colourants, fillers, 
stabilisers, catalysts, biocides and flame retardants and at concentrations of up to several per cent on a dry weight basis [10]. While most additives are designed to be non-migratable from the virgin matrix, leaching may be facilitated by ageing and abrasion of the solid [9], or the very conditions that marine litter are subjected to during transport in sea water and subsequent beaching.

The analysis of metals in manufactured and processed solids by wet chemical means, involving the complete digestion of the material in concentrated acid and subsequent analysis by, for example, inductively coupled plasma (ICP), spectrometry is rather time-consuming and labour-intensive. More appropriate for the analysis of beach litter, therefore, is a non-destructive technique that allows the rapid throughout of samples of diverse composition and that can be used to explore the spatial distribution of metals within a single- or multiple-component sample. With the miniaturisation of $\mathrm{x}$-ray sources, reduction in battery power requirements, and improvements in detector resolution, detection limits, precision and standardless calibrations, field-portable-Xray fluorescence (FP-XRF) spectrometry meets these needs [11].

Nakashima et al. [9] recently employed energy dispersive FP-XRF spectrometry (alpha-6500, Innov-X) to determine the concentrations of five metals-metalloids (As, $\mathrm{Cr}, \mathrm{Pb}, \mathrm{Sb}, \mathrm{Sn})$ in a number of plastic items retrieved from a beach in south west Japan. Measurements were corrected using element-specific and material-specific regression equations that related XRF measurements to those returned by ICP after $\mathrm{HNO}_{3}-\mathrm{H}_{2} \mathrm{SO}_{4}$ digestion. Unfortunately, however, data reported in the article were limited to mean concentrations of $\mathrm{Pb}$ in polyethylene, polypropylene and polyvinyl chloride (PVC) and a mean concentration of Sn in PVC. Moreover, little information 
was provided on the XRF methodology itself, including counting time, fluorescent Xray energy ranges, potential interferences, thickness considerations, and the mode and internal calibration of the instrument.

In the present paper, we describe the use of a Niton FP-XRF spectrometer to determine the concentration of a larger suite of elements $(n=17)$ in a wide variety of samples of marine litter (including plastics, rubbers, expanded-extruded materials, processed corks, ropes, netting and painted surfaces) retrieved from four different beaches. We use the instrument in a 'plastics' mode that is specifically configured for the analysis of complex, low density materials, and evaluate the effects of sample thickness, density and heterogeneity and various aspects of quality control, including a comparison with independent ICP measurements based on analysis of acid digests. The advantages and limitations of the XRF technique used both in situ and ex situ are also discussed.

\section{Materials and methods}

\subsection{Sampling and sample locations}

Four beaches in south west England were visited just after high water on single occasions between late August and late September, 2015. Saltram beach is a small, sandy, intertidal region on the east bank of a shallow, urbanised estuary (Plym) that is close to a recently capped landfill site. Mount Batten is a south-facing pebble-sand beach in Plymouth Sound, a bay that receives freshwater inputs from the Plym and Tamar estuaries and, being protected to the south by a $1.6 \mathrm{~km}$ breakwater, is a natural harbour for naval and commercial ships and a popular location for yachting. 
123 Constantine Bay and Porth Kidney are sandy beaches on the north (Atlantic) coast of

124 Cornwall and are popular destinations for both tourists and surfers.

126 On each beach, visible pieces of litter were collected by hand from a transect of the

127 high (or highest) water line. The length of each transect varied between about $10 \mathrm{~m}$

128 and $200 \mathrm{~m}$ depending on the abundance of material (and ensuring that at least 50

129 samples were collected in each case). Litter retrieved included whole objects and

130 fragments of plastic and rubber, pieces of low density expanded-extruded plastic and

131 rubber and fragments of processed cork (the latter being visually very similar to many

132 expanded polymers), offcuts of rope, fabric and netting, and painted wood, fibreglass

133 and synthetic resin; hereafter, these components are classified as plastics, foams,

134 ropes and painted surfaces, respectively. Note that litter not considered in the present

135 study included metal objects, pieces of glass, paper items, unpainted wood and films

136 of food packaging that appeared to have been recently discarded in situ.

138 Litter from each beach was stored in a series of clear polyethylene bags and

139 transported to the laboratory where individual items were cleared of sand and other

140 debris under running tap water and with the aid of a Nylon brush before being dried at

$14140{ }^{\circ} \mathrm{C}$ in an oven for $12 \mathrm{~h}$ or, for foams, under desiccation at room temperature for up

142 to $96 \mathrm{~h}$. Samples were then weighed on a five-figure balance and stored individually

143 in labelled polyethylene specimen bags and in the dark pending analysis.

\subsection{XRF analysis}

146 Samples of litter were analysed by energy dispersive FP-XRF using a battery-

147 powered, field portable (1.3 kg) Niton XRF analyser (model XL3t $950 \mathrm{He}$ GOLDD+). 
148 The instrument employs a miniature, low power x-ray tube with an Ag transmission

149 anode operating at up to $50 \mathrm{kV}$ of high voltage and $200 \mu \mathrm{A}$ of current as the source of

150 sample excitation, and is fitted with a geometrically optimised large area silicon drift

151 detector to detect and register characteristic $\mathrm{x}$-rays from the sample. The

152 concentrations of As, $\mathrm{Ba}, \mathrm{Bi}, \mathrm{Br}, \mathrm{Cd}, \mathrm{Cl}, \mathrm{Cr}, \mathrm{Cu}, \mathrm{Fe}, \mathrm{Hg}, \mathrm{Ni}, \mathrm{Pb}, \mathrm{Sb}, \mathrm{Se}, \mathrm{Sn}, \mathrm{Ti}$ and

$153 \mathrm{Zn}$, whose fluorescent peaks range from $2.62 \mathrm{keV}\left(\mathrm{Cl}-\mathrm{K}_{\alpha}\right)$ to $32 \mathrm{keV}\left(\mathrm{Ba}-\mathrm{K}_{\alpha}\right)$, were

154 determined in a plastics mode through a fundamental parameters-based alpha

155 coefficient correction model. This iterative approach accounts for background matrix

156 effects by describing measured fluorescent $\mathrm{x}$-ray intensities using mathematical

157 equations that tie together the physics of the interaction of $\mathrm{x}$-rays with sample

158 components [12]. Fundamental parameters eliminates the requirement for sample-

159 specific standards, has a wide dynamic range and is independent of the size and shape

160 of the surface [11].

161

162 Because polymers are composed of light elements, and primarily carbon and

163 hydrogen, they are weak absorbers of x-rays, and below a critical, saturation

164 thickness, $d_{\text {sat }}$, the measured intensity of x-rays will be dependent on both the analyte

165 concentration and the thickness of the sample, $d$. For polyethylene, $d_{\text {sat }}$, is about 9

$166 \mathrm{~mm}$, while for polyvinyl chloride (PVC) the abundance of chloride results in a $d_{\text {sat }}$ of

167 about $5 \mathrm{~mm}$. For polymers containing heavier elements as, for example, contaminants

168 and additives, $d_{\text {sat }}$ is reduced compared with that of the pure material, while in

169 expanded-extruded synthetic polymers, ropes and corks, the presence of air results in

170 densities that are reduced compared with those of the corresponding unexpanded or

171 air-free equivalents and $d_{\text {sat }}$ is increased. For the analysis of polymeric materials in the

172 plastics mode, the Niton XL3t 950 GOLDD+ has a thickness correction algorithm 
173 down to $0.05 \mathrm{~mm}$ that employs a compensation for mass absorption coefficient based

174 on Compton scatter so that variations in density are factored in. In practice, the

175 thickness of each sample needs to be measured (in $\mathrm{mm}$ ) before being analysed and if

176 it falls below $d_{\text {sat }}$, the corrective algorithm should be applied with the appropriate

177 (measured) thickness.

178

179 In the present study, thickness correction was employed for all plastic samples of $d<$

$18010 \mathrm{~mm}$, including those identified by the XRF as PVC (based on $\mathrm{Cl}$ content), and to

181 expanded-extruded polymers, ropes and corks of $d<100 \mathrm{~mm}$ (effectively all samples

182 in these categories). Thickness itself was determined through the flattest

183 ('measurement') surface using $300 \mathrm{~mm}$ Allendale digital callipers, and to increase the

184 effective depth and flatness of thin or hollow samples, items were often cut (with

185 scissors, pliers or a blade), folded or layered and, where necessary, held in place using

186 crocodile clips. Regarding the painted surfaces, a thickness correction of $0.05 \mathrm{~mm}$ was

187 applied in order to ensure that measurements were made of the surface film while

188 minimising interferences from the underlying wooden or polymeric substrates.

189

190 The XRF was used in the laboratory in a bench top accessory stand (with the nose

191 upwards) and was connected to a laptop computer via USB and a remote trigger.

192 Samples were placed on to a SpectraCertified Mylar polyester 3.6 $\mu \mathrm{m}$ film with the

193 measurement surface facing downwards. The slide was then positioned such that the

194 sample lay directly and centrally over a $3 \mathrm{~mm}$ small-spot collimator above the $8 \mathrm{~mm}$

195 XRF measurement window, a process aided by referring to real-time video footage

196 generated by an integrated CCD camera adjacent to the detector. On closing the steel

197 shield of the stand, measurements with appropriate thickness correction were 
activated through the laptop for a total period of 120 seconds; specifically, counting was performed for 60 seconds each in a low energy range $(20 \mathrm{kV}$ and $100 \mu \mathrm{A}: \mathrm{Cl}, \mathrm{Cr}$ and $\mathrm{Ti}$ ) and main energy range (50 kV and $40 \mu \mathrm{A}$ : all remaining elements). Spectra were quantified by fundamental parameter coefficients to yield elemental concentrations on a dry weight basis (in $\left.\mu \mathrm{g} \mathrm{g}^{-1}\right)$ and with a counting error of $2 \sigma(95 \%$ confidence). At the end of each 2-6 h sample measurement session, spectra and

204 elemental concentrations were downloaded to the laptop using Niton data transfer 205 (NDT) PC software.

\subsection{Sample digestion and analysis by ICP}

208 As an independent and more sensitive measure of the elemental content of marine

209 litter, a variety of samples $(n=18)$, encompassing all material categories with the 210 exception of painted surfaces and that had already been tested by XRF, were digested

211 in Fisher Scientific TraceMetal grade acids (and ammonia) and analysed by

212 inductively coupled plasma-optical emission spectroscopy (ICP-OES). Thus, offcuts

213 or slices of plastic, foam (including cork) and rope of between about $50 \mathrm{mg}$ and 200

214 mg were prepared with a stainless steel blade before being accurately weighed into 215 individual $100 \mathrm{ml}$ borosilicate Tecator tubes. Three $\mathrm{ml}$ of concentrated sulphuric acid 216 was added to each tube and the contents heated to $300^{\circ} \mathrm{C}$ for about $2 \mathrm{~h}$ in an 217 aluminium digestion block. To the resulting digests, concentrated $\mathrm{HNO}_{3}$ was carefully 218 added dropwise until the solutions changed from black to pale yellow or colourless.

219 After the covered contents had been allowed to cool overnight, digests were 220 transferred to $50 \mathrm{ml}$ glass volumetric flasks and diluted to mark with $2 \% \mathrm{HNO}_{3}$. To 221 any digests containing a visible precipitate (presumably $\mathrm{PbSO}_{4}$ and/or $\mathrm{BaSO}_{4}$ ), 
concentrated ammonia solution was added dropwise until the solids cleared.

223 Procedural controls were undertaken likewise but in the absence of litter samples.

225 Digests were analysed for all elements detected by XRF and that were measurable by

226 ICP-OES (thereby excluding $\mathrm{Cl}$ and $\mathrm{Br}$ ) using a Thermoscientific iCAP 7400. The 227 instrument was calibrated using four mixed standards and a blank prepared by serial 228 dilution of CPI International standards in $2 \% \mathrm{HNO}_{3}$, and settings in both UV and 229 visible light were as follows: exposure time $=2 \mathrm{~s}$; RF power $=1150 \mathrm{~W}$; nebuliser, 230 coolant and auxiliary gas flows $=0.50,12$ and $0.5 \mathrm{~L} \mathrm{~min}^{-1}$, respectively; viewing 231 height $=12 \mathrm{~mm}$; uptake time $=50 \mathrm{~s} ;$ wash time $=15 \mathrm{~s}$.

2.4. FTIR analysis

234 In order to identify the component polymers in the plastics, foams and ropes, selected 235 samples $(n=50)$ were analysed by attenuated total reflection-Fourier transform infra236 red spectroscopy (ATR-FTIR) using a Bruker ALPHA Platinum ATR QuickSnap 237 A220/D-01 spectrometer. Samples were cut to a suitable size, where necessary, using 238 a stainless steel scalpel, and then firmly clamped down on to the ATR diamond crystal 239 in order to ensure good contact and sufficient penetration of the evanescent wave.

240 Measurements, consisting of 16 scans in the range 4000 to $400 \mathrm{~cm}^{-1}$ and at a

241 resolution of $4 \mathrm{~cm}^{-1}$, were activated via Bruker OPUS spectroscopic software, with 242 subsequent identification involving comparisons of transmittance spectra with 243 libraries of reference spectra.

\section{3. Results and Discussion}

246 3.1. Sample characteristics 
247 A total of 376 litter samples retrieved from four beaches of south west England were

248 analysed in the present study, of which 149 were categorised as plastics, 149 as foams

249 (including processed corks), 68 as ropes and 10 as painted surfaces. The mass of

250 individual items of plastic ranged from less than $10 \mathrm{mg}$ for various unidentifiable

251 fragments to over $10 \mathrm{~g}$ for relatively large fragments and specific objects (e.g. toys, a

252 bodyboard clip and a cigarette lighter); thickness through the flattest surface, $d$,

253 ranged from about $0.3 \mathrm{~mm}$ for several flexible films after folding or stacking to more

254 than $10 \mathrm{~mm}$ for many of the largest fragments and objects. The mass of the foams

255 ranged from less than $3 \mathrm{mg}$ to $50 \mathrm{~g}$ and $d$ ranged from about 0.5 to $40 \mathrm{~mm}$, with most

256 items being fragments of various size, shape and colour (but mainly off-white or

257 yellow-brown). The mass of ropes ranged from about $20 \mathrm{mg}$ for a small fragment of

258 fabric to over $40 \mathrm{~g}$ for a multi-coloured offcut of rigging, with $d$, after any deliberate

259 intertwining of fishing line or frayed netting, ranging from about 2 to $12 \mathrm{~mm}$. The

260 mass of painted surfaces ranged from about 1.7 to $150 \mathrm{~g}$, with the bulk of the material

261 representing the underlying substrate, and in all cases $d$ (of the surface) was assumed

262 to be $0.05 \mathrm{~mm}$ (see above).

264 FTIR analysis of selected samples revealed that plastic items and fragments were

265 mainly polyethylene or polypropylene but a few were polyvinyl chloride (PVC), and

266 that offcuts of rope and netting were generally polyethylene or polypropylene but

267 fishing lines were Nylon. Many pieces of expanded-extruded polymer could not be

268 identified definitively but those yielding clear spectra were predominantly polystyrene

269 or polyurethane with some fragments composed of elasticated rubber, PVC, rayon or

270 acrylic. Because information on cork was not contained in the reference libraries, such 
samples were verified by comparisons of spectra with those derived from direct measurements of a series of cork stoppers.

\subsection{Detection limits}

275

FP-XRF is able to detect elements that elicit a sufficiently strong fluorescence signal relative to background intensity that is free from interferences arising from the matrix and from the interactions of other elements (peak overlaps and absorption and enhancement effects). The limit of detection (LOD) is, therefore, dependent on the element (or its X-ray activity and the energies of its fluorescence peaks), detector resolution, mode of application of the instrument, counting time, sample thickness, physical and chemical composition of the material, and statistical criteria used for defining detection. The Niton XLT3t series of analysers define LODs that are specific to the characteristics of the sample and the counting time as three standard deviations ( $1.5 \times 2 \sigma$, or $99.7 \%$ confidence interval), and measurements are reported in the NDT output only where concentrations exceed this threshold with calculated detection limits provided otherwise. The lower LODs specified by the manufacturer for elements in 'clean' polymers of unspecified thickness that are relatively free of interelement spectral interferences and for a 30-second total counting time in plastics mode range from less than $10 \mu \mathrm{g} \mathrm{g}^{-1}$ ( $\mathrm{As}, \mathrm{Bi}, \mathrm{Br}, \mathrm{Hg}, \mathrm{Pb}$ and $\mathrm{Se}$ ) to $100 \mu \mathrm{g} \mathrm{g}^{-1}$ (Ba) in polyethylene and from less than $20 \mu \mathrm{g} \mathrm{g} \mathrm{g}^{-1}$ (As, $\mathrm{Br}, \mathrm{Cd}$ and $\mathrm{Pb}$ ) to more than $100 \mu \mathrm{g} \mathrm{g}^{-1}$ (Fe) in PVC.

LODs based on the analysis of the more varied and complex litter samples are shown in Table 1. Here, the median, minimum and maximum calculated limits for each element and for each category of sample are provided for a total counting time of 120 
seconds and with appropriate thickness correction where applicable (see below), along with the numbers and percentages of samples that were not detected in each case. Within a specific material category, LODs vary widely for a given element, reflecting the sensitivity of detection to sample composition and thickness and the presence of

300 and interferences arising from other elements in the matrix. For most elements, and

301 within a given category, LODs span one or two orders of magnitude among the samples. With respect to the different material categories, and based on median values, LODs are higher in foams than in plastics and ropes, presumably because of the greater contribution of air in the former (air promotes multilateral reflections and inhibits fluorescent x-rays from reaching the detector [13]). Painted surfaces exhibit 306 the lowest LODs, likely because samples in this category contained fewer elements and lower concentrations of $\mathrm{Cl}$ as potential interferents (attenuating or enhancing secondary x-rays). Among the elements, LODs were generally lowest, and below 40 $\mu \mathrm{g} \mathrm{g}{ }^{-1}$ for each category of material, for $\mathrm{As}, \mathrm{Br}, \mathrm{Cr}$ and $\mathrm{Pb}$, and highest, and above 70

$310 \mu \mathrm{g} \mathrm{g}^{-1}$ for each category, for $\mathrm{Ba}$ and $\mathrm{Cl}$.

\subsection{Thickness correction and reference materials}

313 Table 2 shows the results of multiple analyses $(n=5)$ of two reference materials manufactured by Niton (PN 180-554, batch SN PE-071-N, and PN 180-619, LOT\#T-

315 18); these are polyethylene discs of $31 \mathrm{~mm}$ in diameter impregnated with various 316 elements and whose thicknesses $(d \sim 13 \mathrm{~mm})$ are above the saturation level for the 317 material. Thus, without thickness correction, the FP-XRF delivers concentrations that 318 are usually within $10 \%$ of the respective reference values; for comparison, an 319 'acceptable' difference from certified values of $\pm 20 \%$ for each analyte is stipulated by the US Environmental Protection Agency (EPA) [14]. Also shown in Table 2 are 
321 results of analyses of a $3.4 \mathrm{~mm}$ section of PN 180-619 with appropriate thickness

322 correction applied. Measured concentrations are within $12 \%$ of corresponding

323 concentrations returned by analysis of the whole disc without thickness correction

324 with the exception of $\mathrm{Ba}$, whose measured concentration in the section was $40 \%$

325 greater than that in the whole disc.

327 Figure 1 illustrates the effect of varying the thickness correction on the concentrations

328 returned, and after normalisation to the measured concentrations without thickness

329 correction (and as annotated), for reference material PN 180-554; these results

330 illustrate how different elements respond to the corrective algorithm in polyethylene.

331 Thus, for $\mathrm{Cr}$ and to a lesser extent $\mathrm{Hg}$, adjusting thickness correction towards its lower 332 limit of $0.05 \mathrm{~mm}$ results in progressively lower normalised concentrations, while for

333 Cd the same adjustments result in a progressively higher concentrations; normalised

334 concentrations of $\mathrm{Br}$ and $\mathrm{Pb}$, on the other hand, exhibit relatively little dependence on

335 the magnitude of the thickness correction applied. Despite different concentrations of

336 several elements being returned that were dependent on the thickness correction

337 applied, relative error did not display any clear dependence on this variable.

339 3.4. Thickness correction and plastic litter

340 Figure 2 exemplifies the effects of applying different thickness corrections to four

341 plastic (polyethylene and polypropylene) samples of varying size, colour, condition-

342 age and elemental composition and where $d<d_{\text {sat. }}$ Here, a wider range of elements

343 detected are normalised to the corresponding concentrations measured after applying

344 correction for the measured thickness, and whose values are annotated on each panel.

345 For a given element, the precise effects and trends are different, both among the 
samples and from the reference material (Figure 1), presumably because of the interactive effects that the presence of additional elements have on each other in more complex materials and the consequent corrections applied by fundamental parameters.

349 In general, however, and with adjustments in thickness correction towards the lower

350 limit, $\mathrm{Ba}$ and $\mathrm{Sb}$ are subject to a significant increase in normalised concentration

351 while $\mathrm{Cl}, \mathrm{Cu}, \mathrm{Fe}, \mathrm{Ti}$ and $\mathrm{Zn}$ exhibit a decrease in normalised concentration. Chlorine 352 exhibited the greatest dependency on thickness correction in respect of the latter, and 353 among the samples $\mathrm{Cu}, \mathrm{Fe}$ and $\mathrm{Zn}$ exhibited a dependency whose significance 354 appeared to exhibit an inverse dependency on the measured concentration with 355 appropriate correction applied. Bromine, $\mathrm{Cd}, \mathrm{Pb}$ and $\mathrm{Se}$ exhibited a dependency on 356 thickness correction that was relatively small, with concentration differences across 357 the range of corrections that varied by no more than $20 \%$. As with the reference 358 materials, relative errors did not display any clear dependence on thickness correction, 359 but were inversely related to the absolute concentration of the element in the sample.

\subsection{Thickness correction and foam and rope litter}

362 Figure 3 shows the effects of applying different thickness corrections to six different 363 samples containing air within their structures; namely, an expanded polystyrene, two 364 expanded, rigid polyurethanes, a Nylon fishing line that had been intertwined and 365 secured between two crocodile clips, a fragment of cork and on offcut of multi366 coloured rope. Because $d_{\text {sat }}$ is unknown and, likely, highly variable among such 367 samples, sub-saturation was assumed in all cases. Thus, as above, the concentrations 368 of different elements detected are normalised to the corresponding concentrations 369 measured after applying correction for the measured thickness, and whose values are 370 annotated on each panel. The findings are broadly consistent with those ascertained 
371 from analyses of the plastics (Figure 2) in that adjustments in thickness correction

372 towards the lower limit of $0.05 \mathrm{~mm}$ are accompanied by an increase in normalised

373 concentration of $\mathrm{Ba}$ (note that $\mathrm{Sb}$ was not detected in these samples) and a reduction

374 in normalised concentration for all other elements detected. The latter effect was most

375 significant for $\mathrm{Cl}, \mathrm{Fe}$ and $\mathrm{Zn}$ and least evident for $\mathrm{Br}$ and $\mathrm{Pb}$, and, for the cork and two

376 of the expanded polymers, was always most pronounced at thickness corrections

377 below 2-6 $\mathrm{mm}$ such that trends were approximately asymptotic to unit value.

\subsection{Measurement precision and sample heterogeneity}

380 Regular assessment of precision is recommended by the US EPA [14] when using FP-

$381 \mathrm{XRF}$ as an important component of quality control considerations. The precision of 382 concentrations delivered by the Niton XL3t 950 He GOLDD+ on beached marine 383 litter was ascertained on a wide range of samples, in terms of material category, size, 384 thickness, colour, condition and elemental content, that were fixed in position relative 385 to the detector and measured repeatedly ( $n=4$ to 6$)$ with appropriate thickness 386 correction. Precision was defined as the relative standard deviation (as a percentage) 387 arising from the repeat measurements of each sample, and results are shown in Table 388 3. Precision was highly variable, both among the different elements and between the 389 different material categories, but in all cases was better than $30 \%$ and in most cases 390 better than 20\%, a recognised 'acceptable' objective for FP-XRF measurements [15].

391 Precision displayed a dependency on absolute concentrations (i.e. measurements of 392 higher elemental concentrations were more precise) and was also dependent on the 393 characteristics of the sample; specifically, measurements were most precise for 394 relatively thick, dense and homogeneous materials and least precise for thin, 395 expanded-extruded and more heterogeneous items. 
397 When the same sample of plastic was located in different positions or with different 398 orientations above the detector, repeat measurements became more variable, reflecting 399 the different response of the detector to variations in geometry and thickness and to 400 inherent variability in the composition and surface conditions of the polymer. Samples 401 composed of regions of visibly different colour or texture or with areas of obvious 402 discolouration, and in particular those in the foams and painted surfaces categories, 403 often exhibited considerable variations in elemental concentrations that reflected 404 significant compositional changes and/or heterogeneous contamination. Figure 4 405 exemplifies the spatial, surface variability of various elements on a piece of painted 406 fibreglass backed with resin that appeared to have been derived from a boat, while 407 Figure 5 illustrates both the surface and depth distribution of different elements in a 408 fragment of cork that had evidently been treated or contaminated. Note an order of 409 magnitude difference in concentrations of $\mathrm{Bi}, \mathrm{Cl}$ and $\mathrm{Pb}$ among the different colours 410 of paint and two orders of magnitude differences in Fe and $\mathrm{Zn}$ concentrations in the 411 different regions of cork.

\subsection{Summary of elemental concentrations in marine litter}

414 Table 4 summarises the concentrations of the elements that were detected in each

415 material category of beached marine litter, with appropriate thickness correction 416 applied. Note that the lowest concentrations reported here for some elements are 417 lower than minimum LODs given in Table 1 because detection is dependent on the 418 precise composition and thickness of the sample and the presence of interfering 419 elements; thus, for example, what may not be detected in one sample may be detected 420 at a lower concentration in another sample which is thicker, denser and 'cleaner'. 
421 Note also that, in the case of As, overlap of its $\mathrm{K}_{\alpha}$ fluorescence peak with the $\mathrm{L}_{\alpha}$ peak

$422 \mathrm{O} \mathrm{Pb}$ and the relatively low intensity of the $\mathrm{As}-\mathrm{K}_{\beta}$ line means that As concentrations

423 cannot be effectively calculated for samples with $\mathrm{Pb}$ :As ratios in excess of about 10

424 [14]. Because false positives may often be returned for the metalloid by FP-XRF in

425 the presence of $\mathrm{Pb}$ [16], As concentrations given in Table 4 are restricted to those

426 returned where $\mathrm{Pb}$ was not detectable.

428 In the plastics category, all elements were detected, albeit in less than ten cases for

429 As, Bi, Cd, Hg, Sb, Se, Sn and Ni. Among the remaining elements, concentrations

430 spanned at least an order of magnitude, and in the case of $\mathrm{Cl}, \mathrm{Fe}, \mathrm{Pb}$, $\mathrm{Ti}$ and $\mathrm{Zn}$, at

431 least three orders of magnitude. Regarding $\mathrm{Cl}$, two samples whose concentrations

432 exceeded $350,000 \mu \mathrm{g} \mathrm{g}^{-1}$ (or $35 \%$ by weight) were returned as PVC by the XRF, an

433 identification that was confirmed by subsequent FTIR analysis. Bismuth, $\mathrm{Cd}, \mathrm{Hg}$ and

434 Se were never detected in the foams but many of the remaining elements returned a

435 higher number (and proportion) of detectable cases in this category compared with the

436 plastics. For example, $\mathrm{Br}, \mathrm{Pb}$ and $\mathrm{Sn}$ were detectable in about 15,13 and $2 \%$ of plastic

437 samples compared with respective percentages of 75, 20 and 14\%, of foam samples.

438 Median concentrations were about an order of magnitude greater in foams than in

439 plastics for $\mathrm{Br}, \mathrm{Cr}, \mathrm{Fe}$ and $\mathrm{Zn}$; for $\mathrm{Cl}$, the difference was more than two orders of

440 magnitude and 35 samples were returned with concentrations in excess of $150,000 \mu \mathrm{g}$

$441 \mathrm{~g}^{-1}$ (or $15 \%$ by weight) and identified as PVC by the XRF. Clearly, many of these

442 samples were not PVC-based (most of those analysed by FTIR were identified as

443 expanded-extruded polystyrene or polyurethane) but such a classification based on a

444 high chlorine content is a practical requirement for the appropriate fundamental

445 parameter coefficients to be applied. 
447 In the ropes category, $\mathrm{Ba}, \mathrm{Bi}, \mathrm{Cd}, \mathrm{Hg}, \mathrm{Sb}$ and $\mathrm{Se}$ were never detected and median 448 concentrations of $\mathrm{Ni}$ and $\mathrm{Pb}$ were higher and $\mathrm{Br}$ and $\mathrm{Zn}$ lower than the respective 449 median concentrations in both plastics and foams; however, compared with the latter 450 categories, concentrations of most detectable elements in ropes encompassed 451 considerably lower ranges in concentrations. Analysis of the painted surfaces returned 452 no detectable $\mathrm{Cd}, \mathrm{Cr}, \mathrm{Ni}, \mathrm{Sb}, \mathrm{Se}, \mathrm{Sn}$ and As and median concentrations of $\mathrm{Ba}$ and $\mathrm{Ti}$ 453 that were higher and $\mathrm{Cl}, \mathrm{Cu}$ and $\mathrm{Fe}$ lower than respective median concentrations in the 454 plastics, foams and ropes categories. With the exception of $\mathrm{Pb}$ and $\mathrm{Zn}$, the range of 455 elemental concentrations in the painted surfaces was lower than in any of the 456 remaining categories. Overall, and for all categories, $\mathrm{Cl}, \mathrm{Fe}$ and $\mathrm{Ti}$ were detected in 457 the most $(>300)$ and $\mathrm{Hg}$ and $\mathrm{Se}$ in the least $(\leq 3)$ number of cases; maximum concentrations exceeded $10,000 \mu \mathrm{g} \mathrm{g}^{-1}$ (or $1 \%$ by weight) for $\mathrm{Ba}, \mathrm{Br}, \mathrm{Cl}, \mathrm{Fe}, \mathrm{Pb}, \mathrm{Ti}$

459 and $\mathrm{Zn}$ but were never greater than $500 \mu \mathrm{g} \mathrm{g}^{-1}$ for $\mathrm{Bi}$ and $\mathrm{Hg}$.

462 As an independent measure of the elemental content of beached litter, selected 463 samples were analysed for all elements except $\mathrm{Br}$ and $\mathrm{Cl}$ by ICP-OES following $464 \mathrm{H}_{2} \mathrm{SO}_{4}-\mathrm{HNO}_{3}$ digestion. Samples encompassed a variety of plastic fragments, expanded polymers (including polyurethane and polystyrene), ropes and a piece of 466 cork but excluded painted surfaces. (Note that while paints could not be completely 467 isolated from their substrates, a comparison of discrete paint fragment analysis by FP$468 \mathrm{XRF}$ and ICP given elsewhere reveals good agreement between the two approaches 469 [16].) The results of the present study revealed no false negatives among the samples 470 and for all elements considered; that is, lack of detection by the XRF was not 
accompanied by a measurement by ICP that exceeded the corresponding LOD of the

XRF. Excluding the impacts of $\mathrm{Pb}$ on the detection of As by XRF (see above), false positives were returned in three cases for $\mathrm{Cr}$ (two plastic fragments and a piece of polystyrene foam); thus, here, the XRF reported concentrations of $\mathrm{Cr}$ (albeit less than $100 \mu \mathrm{g} \mathrm{g}^{-1}$ ) in samples that failed to return a measurement by the more sensitive ICPOES.

Concentrations of various elements detected by both approaches are compared in

Figure 6 and statistical summaries for the data and for individual elements are shown in Table 5. Correlation analysis revealed significant relationships between [XRF] and [ICP] for all elements with the exception of $\mathrm{Ba}$, and slopes, when forced through the origin, of less than 1.4 with the exception of $\mathrm{Cu}$ (about 8), Sn (4) and $\mathrm{Zn}$ (2.5) 2.5 and 1.8 , respectively). Despite most slopes being greater than 1 , however, or the XRF on average returning concentrations greater than those delivered by the ICP, the relationship defining the data overall and forced through the origin $(y=0.850 x ; r=$ $0.775 ; p<0.01 ; n=89)$ indicates a slope below unit value. This discrepancy may be attributed to the weighting placed on the two data points defining the highest concentrations of Fe; thus, neglecting these points results in a slope of 1.24 ( $r=0.858$; $p<0.01 ; n=91)$.

492 Given that FP-XRF provides a measurement of the elemental content at a particular 493 location on the sample whereas ICP involves complete destruction of a sample (that 494 likely displays some degree of heterogeneity), coupled with possible incomplete 495 digestion of some samples on acid treatment and the presence of precipitate in other 
cases, the two approaches could be considered as providing more than acceptable agreement overall according to EPA criteria [14]. Based on the results for individual elements, the quantitative performance of the XRF appears to be considerably better

499 for elements like $\mathrm{Cd}, \mathrm{Pb}, \mathrm{Sb}$ and $\mathrm{Se}$ than for $\mathrm{Cu}, \mathrm{Sn}$ and $\mathrm{Zn}$, and in particular in 500 expanded-extruded materials where accurate thickness correction is critical.

\subsection{Advantages and limitations of FP-XRF for the analysis of marine litter}

FP-XRF affords a cost-effective, non-destructive means of measuring elements in a variety of materials sampled from the pool of litter washed up on beaches, including plastics and rubbers, synthetic foams, corks, painted surfaces, ropes, fabrics and netting. With respect to the Niton XL3t 950 He GOLDD+, because it does not employ a radioactive source for the excitation of samples, training, usage and transport are not constrained by additional safety considerations and licensing and its excitation source is not subject to regular replacement. In the plastics mode, the instrument is able to

510 analyse samples with very little preparation down to a few $\mathrm{mg}$ in weight and less than

$5113 \mathrm{~mm}$ in diameter, including plastic production pellets, individual spheres of expanded 512 polystyrene, and small fragments of (micro)plastic, foam and rope, with a minimum

513 LOD of less than $10 \mu \mathrm{g} \mathrm{g}^{-1}$ and a precision of better than $10 \%$ in many cases. The 514 instrument is particularly useful for measuring the spatial and depth distributions of 515 elements in larger items and on heterogeneous surfaces which exhibit variable degrees 516 of contamination.

518 Although samples whose thickness is greater than saturation are preferable in that the 519 analysis is more sensitive and returns relatively low counting errors, an algorithm in 520 the plastics mode is able to correct for measurements made on thinner samples in 
most cases. While sample thickness has a relatively small impact on concentrations returned for some elements, like $\mathrm{Br}, \mathrm{Pb}$ and $\mathrm{Se}$, it has a significant impact on measurements of others, including $\mathrm{Ba}, \mathrm{Cl}, \mathrm{Cr}, \mathrm{Cu}$, $\mathrm{Ti}$ and $\mathrm{Zn}$, and particularly in materials containing air, like expanded-extruded polymers and ropes, and whose thickness is below a few mm. It is critical, therefore, to apply the corrective algorithm

526 if the latter elements are considered in samples comprised of low density materials and where $d<<d_{\text {sat. }}$.

An additional advantage of FP-XRF, and which has been reported in practice in the

530 literature, is the ability to test beach litter samples in situ [9]. Pilot studies undertaken

531 with the Niton XL3t 950 He GOLDD+ on one beach involved activating the

532 instrument through the touch screen display in order to measure samples in contact

533 with or in close proximity to the detector window and that had been temporarily fixed

534 to a white plastic tray using adhesive putty. While this approach proved useful for the 535 relatively rapid screening of some elements and the approximate abundance of others, 536 detection limits and counting errors were relatively high and precision relatively low. 537 This is because of the shorter counting time employed in our pilot study (20 seconds 538 each in the main and low energy ranges), hand-held vibrations of the XRF nose and 539 inconsistent positioning of samples in front of the detector window.

541 In situ application of the FP-XRF was also subject to practical difficulties associated 542 with accurately measuring sample thickness, cleaning, drying and cutting-folding 543 samples, orientating the flattest surface towards the detector, and measuring samples 544 smaller than the diameter of the measurement window (i.e. $<8 \mathrm{~mm}$ ); for a single 545 operator of the instrument, these difficulties had a significant impact on the 
546 throughput of samples. Safety was an additional concern since the analysis of

547 irregular surfaces or shapes of low density tends to create excess secondary (back

548 scattered and fluorescent) radiation. Given these constraints and considerations, and

549 our requirements for weighing, archiving and additional testing, a laboratory approach

550 involving a customised stand is recommended for the more accurate and systematic

551 characterisation of beached marine litter.

552

553 From an environmental perspective, the most important finding of the present study

554 was the occurrence and elevated concentrations of hazardous elements in many

555 samples and from all material categories considered. Most significant in this respect

556 are the heavy metals, $\mathrm{Cd}$ and $\mathrm{Pb}$, at concentrations in excess of $1000 \mu \mathrm{g} \mathrm{g}^{-1}$ in various

557 plastics objects and fragments, $\mathrm{Pb}$ and $\mathrm{Br}$ (the latter a component of brominated flame

558 retardants) up to concentrations of $2 \%$ in expanded-extruded polymers, and $\mathrm{Pb}$ at

559 concentrations of several thousand $\mu \mathrm{g} \mathrm{g}^{-1}$ in fragments of rope, netting and processed

560 cork and in paint used on wood and fibreglass. The mobilities and bioaccessibilities of

561 these elements are important considerations for risk assessment and management

562 purposes and are the subject of ongoing research.

563

564 Acknowledgements

565 We are grateful to Dr Andy Fisher, UoP, for performing the digests of the litter

566 samples, and Dr Ken Grainger, Niton UK, for discussions and advice relating to the

567 XRF analyses. This study was funded partly by a UoP Marine Institute grant.

References 
570 [8] K. Ashton, L. Holmes, A. Turner, Association of metals with plastic production

571 pellets in the marine environment, Mar. Pollut. Bull. 60 (2010) 2050-2055.

573 [11] G.L. Bosco, Development and application of portable, hand-held X-ray

574 fluorescence spectrometers, Trend. Anal. Chem. 45 (2013) 121-134.

575

576 [4] S. Endo, R. Takizawa, K. Okuda, H. Takada, K. Chiba, H. Kanehiro, H. Ogi, R.

577 Yamashita, Concentration of polychlorinated biphenyls (PCBs) in beached resin

578 pellets: Variability among individual particles and regional differences, Mar. Pollut.

579 Bull. 50 (2005) 1103-1114.

580

581 [14] Environmental Protection Agency, Method 6200 - Field portable x-ray

582 fluorescence spectrometry for the determination of elemental concentrations in soil

583 and sediment (2007).

584 http://www3.epa.gov/epawaste/hazard/testmethods/sw846/pdfs/6200.pdf

585 Accessed 12/15.

586

587 [5] J.P.G.L. Frias, P. Sobral, A.M. Ferreira, Organic pollutants in microplastics from

588 two beaches of the Portuguese coast, Mar. Pollut. Bullet. 60 (2010) 1988-1992.

589

590 [6] J. Gauquie, L. Devrese, J. Robbens, B. De Witte, A qualitative screening and

591 quantitative measurement of organic contaminants on different types of marine plastic

592 debris, Chemosphere 138 (2015) 348-356.

593 
594 [10] E. Hansen, N.H. Nilsson, D. Lithner, C. Lassen, Hazardous substances in plastic 595 materials, COWI and the Danish Technological Institute on behalf of The Norwegian 596 Climate and Pollution Agency, Oslo (2010) 150 pp.

598 [7] L.A. Holmes, A. Turner, R.C. Thompson, Adsorption of trace metals plastic resin 599 pellets in the marine environment, Environ. Pollut. 160 (2012) 42-48.

600

601 [15] D.J. Kalnicky, R. Singhvi, Field portable XRF analysis of environmental 602 samples. J. Hazard. Mater. 83 (2001) 93-122.

603

604 [3] Y. Mato, T. Isobe, H. Takada, H. Kanehiro, C. Ohtake, T. Kaminuma, 605 Plastic resin pellets as a transport medium for toxic chemicals in the marine 606 environment, Environ. Sci. Technol. 35 (2001) 318-324.

607

608 [1] G.R. Murray, Environmental implications of plastic debris in marine settings609 entanglement, ingestion, smothering, hangers-on, hitch-hiking and alien invasions, 610 Phil. Trans. Royal Soc. Lond. B: Biol. Sci. 364 (2009) 2013-2025.

611

612 [9] E. Nakashima, A. Isobe, S. Kako, T. Itai, S. Takahashi, Quantification of toxic 613 metals derived from macroplastic litter on Ookushi beach, Japan, Environ. Sci.

614 Technol. 46 (2012) 10099-10105.

615

616 [12] S. Piorek, Feasibility of analysis and screening of plastics for heavy metals with 617 portable x-ray fluorescence analyser with miniature x-ray tube, GPEC 2004 Paper 618 abstract \#14 (2004). 
620 [13] M. Schlummer, J. Vogelsang, D. Fiedler, L. Gruber, G. Wolz, Rapid

621 identification of PS foam wastes containing HBCDD or its alternative PolyFR by x-

622 ray fluorescence spectroscopy (XRF), Waste Manag. Res. (2015)

623 doi:10.1177/0734242X15589783

624

625 [2] K. Tanaka, H. Takada, R. Yamashita, K. Mizukawa, M. Fukuwaka, Y. Watanuki, 626 Facilitated leaching of additive-derived PBDEs from plastic by seabirds' stomach oil 627 and accumulation in tissues. Environ. Sci. Technol. 49 (2015) 11799-11807. 628

629 [16] A. Turner, S. Comber, A.B. Rees, D. Gkiokas, K. Solman, Metals in boat paint 630 fragments from slipways, repair facilities and abandoned vessels: an evaluation using 631 portable XRF, Talanta 131 (2014) 372-378. 
Table 1: Median, minimum and maximum limit of detection (LOD, $\mu \mathrm{g} \mathrm{g}^{-1}$ ) for each element and in the four categories of litter for a total counting time of 120 seconds and with appropriate thickness correction applied. Also shown are the numbers and percentages of cases where elements were not detected in each category and which were used to quantify the LODs. Original res version in Excel file.

\begin{tabular}{|c|c|c|c|c|c|c|c|c|c|c|c|c|c|c|c|c|c|c|c|c|}
\hline & \multicolumn{5}{|c|}{ plastics $(n=149)$} & \multicolumn{5}{|c|}{ foams $(n=149)$} & \multicolumn{5}{|c|}{ ropes $(n=68)$} & \multicolumn{5}{|c|}{ painted surfaces $(n=10)$} \\
\hline & \multicolumn{3}{|c|}{$n<$ LOD $\%<$ LOD median } & \multirow{2}{*}{$\begin{array}{r}\min \\
1.8\end{array}$} & \multirow{2}{*}{$\frac{\max }{231}$} & \multicolumn{3}{|c|}{$n<$ LOD $\%<$ LOD median } & \multirow{2}{*}{$\begin{array}{r}\min \\
2.9\end{array}$} & \multirow{2}{*}{$\begin{array}{r}\frac{\max }{420} \\
\end{array}$} & \multicolumn{3}{|c|}{$n<$ LOD $\%<$ LOD median } & \multirow{2}{*}{$\begin{array}{r}\min \\
2.3\end{array}$} & \multirow{2}{*}{$\begin{array}{r}\frac{\max }{47} \\
4\end{array}$} & \multicolumn{2}{|c|}{$n<$ LOD $\%<$ LOD } & \multirow{2}{*}{$\begin{array}{r}\text { median } \\
1.5\end{array}$} & \multirow{2}{*}{$\begin{array}{r}\min \\
1.5\end{array}$} & \multirow{2}{*}{$\begin{array}{r}\max \\
1.9\end{array}$} \\
\hline As & 143 & 96.0 & 3.4 & & & 138 & 92.6 & 23 & & & 59 & 86.8 & 5.6 & & & 6 & 60 & & & \\
\hline $\mathrm{Ba}$ & 132 & 88.6 & 492 & 217 & 2011 & 140 & 94.0 & 498 & 185 & 1734 & 68 & 100 & 468 & 281 & 912 & 8 & 80 & 384 & 342 & 454 \\
\hline $\mathrm{Bi}$ & 145 & 97.3 & 12 & 5.5 & 66 & 149 & 100 & 49 & 7.1 & 361 & 68 & 100 & 17 & 8.4 & 79 & 9 & 90 & 5.3 & 3.9 & 72 \\
\hline $\mathrm{Br}$ & 126 & 84.6 & 6.7 & 2.9 & 30 & 37 & 24.8 & 36 & 9.9 & 75 & 36 & 52.9 & 7.7 & 4.6 & 21 & 3 & 30 & 14 & 1.8 & 14 \\
\hline $\mathrm{Cd}$ & 144 & 96.6 & 52 & 25 & 147 & 149 & 100 & 57 & 22 & 197 & 68 & 100 & 53 & 33 & 104 & 10 & 100 & 28 & 24 & 38 \\
\hline $\mathrm{Cl}$ & 22 & 14.8 & 100 & 19 & 260 & 6 & 4.0 & 1233 & 468 & 1788 & 5 & 7.4 & 341 & 81 & 459 & 0 & 0 & & & \\
\hline $\mathrm{Cr}$ & 72 & 48.3 & 14 & 2.7 & 46 & 41 & 27.5 & 35 & 8.7 & 305 & 36 & 52.9 & 14 & 4.9 & 31 & 10 & 100 & 1.5 & 1.5 & 1.7 \\
\hline $\mathrm{Cu}$ & 114 & 76.5 & 24 & 7.9 & 123 & 120 & 80.5 & 145 & 17 & 562 & 21 & 30.9 & 34 & 21 & 100 & 8 & 80 & 6.0 & 3.8 & 16 \\
\hline $\mathrm{Fe}$ & 28 & 18.8 & 25 & 15 & 56 & 1 & 0.7 & 625 & 625 & 625 & 1 & 1.5 & 56 & 56 & 56 & 0 & 0 & & & \\
\hline $\mathrm{Hg}$ & 148 & 99.3 & 13 & 6.0 & 109 & 149 & 100 & 68 & 8.7 & 287 & 68 & 100 & 15 & 9.2 & 48 & 8 & 80 & 3.8 & 2.8 & 12 \\
\hline $\mathrm{Ni}$ & 146 & 97.9 & 20 & 8.4 & 173 & 147 & 98.7 & 142 & 21 & 544 & 66 & 97.1 & 31 & 20 & 102 & 10 & 100 & 6.0 & 4.9 & 10 \\
\hline $\mathrm{Pb}$ & 130 & 87.2 & 7.3 & 3.4 & 47 & 120 & 80.5 & 30 & 12.0 & 98 & 40 & 58.8 & 9.1 & 5.4 & 25 & 4 & 40 & 2.8 & 2.5 & 3.8 \\
\hline $\mathrm{Sb}$ & 144 & 96.6 & 85 & 31 & 243 & 145 & 97.3 & 85 & 34 & 326 & 68 & 100 & 82 & 51 & 160 & 10 & 100 & 57 & 47 & 77 \\
\hline $\mathrm{Se}$ & 147 & 98.7 & 11 & 4.8 & 126 & 149 & 100 & 45 & 6.0 & 215 & 68 & 100 & 11 & 7.0 & 34 & 10 & 100 & 3.5 & 2.6 & 14 \\
\hline Sn & 146 & 98.0 & 72 & 34 & 221 & 127 & 85.2 & 76 & 26 & 275 & 67 & 98.5 & 73 & 44 & 133 & 10 & 100 & 37 & 24 & 38 \\
\hline $\mathrm{Ti}$ & 13 & 8.7 & 8.9 & 2.5 & 28 & 6 & 4.0 & 314 & 95 & 1768 & 1 & 1.5 & 16 & 16 & 16 & 0 & 0 & & & \\
\hline $\mathrm{Zn}$ & 76 & 51.0 & 12 & 6.4 & 36 & 60 & 40.3 & 91 & 17 & 238 & 40 & 58.8 & 15 & 10 & 34 & 3 & 30 & 6.1 & 5.1 & 6.4 \\
\hline
\end{tabular}


Table 2: A comparison of reference and measured concentrations $\left(\mu \mathrm{g} \mathrm{g}^{-1}\right)$ in two polyethylene discs supplied by Niton and that had been impregnated with various elements. Note that reference concentrations are given with estimates of errors at the 95\% confidence level arising from an unspecified number of analyses while measured concentrations are shown as the mean with one standard deviation $(n=5)$. Original res version in Excel file.

\begin{tabular}{|c|c|c|c|c|c|c|c|c|c|}
\hline material & As & $\mathrm{Ba}$ & $\mathrm{Br}$ & $\mathrm{Cd}$ & $\mathrm{Cr}$ & $\mathrm{Hg}$ & $\mathrm{Pb}$ & $\mathrm{Sb}$ & $\mathrm{Se}$ \\
\hline \multicolumn{10}{|l|}{ PN 180-554 } \\
\hline reference & & & $495 \pm 20$ & $150 \pm 6$ & $995 \pm 40$ & $1000 \pm 40$ & $1002 \pm 40$ & & \\
\hline measured with no thickness correction & & & $49 \overline{7} \pm 3$ & $135 \pm 5$ & $106 \overline{0}_{ \pm} 7$ & $912 \pm 5$ & $964 \pm 21$ & & \\
\hline \multicolumn{10}{|l|}{ PN 180-619 } \\
\hline reference & $51 \pm 7$ & $704 \pm 45$ & & $292 \pm 20$ & $106 \pm 10$ & $101 \pm 10$ & $155 \pm 12$ & $94+10$ & $207 \pm 15$ \\
\hline measured with no thickness correction & $46 \pm 2$ & $761 \pm 25$ & & $295 \pm 5$ & $116 \pm 8$ & $93 \pm 3$ & $136 \pm 2$ & $98 \pm 3$ & $228 \pm 4$ \\
\hline \multicolumn{10}{|l|}{ PN 180-619: 3.4 mm section } \\
\hline measured with thickness correction & $44 \pm 4$ & $1060 \pm 130$ & & $319 \pm 12$ & $124 \pm 2$ & $82 \pm 5$ & $138 \pm 7$ & $109_{ \pm} 19$ & $209+5$ \\
\hline
\end{tabular}


Table 3: Number of cases detected and median, minimum and maximum precisions (as \% relative standard deviation) for each element and in the four categories of litter for a total counting time of 120 seconds and with appropriate thickness correction applied. Original res version in Excel file.

\begin{tabular}{|c|c|c|c|c|c|c|c|c|c|c|c|c|c|c|c|c|}
\hline & \multicolumn{4}{|c|}{ plastics $(n=6)$} & \multicolumn{4}{|c|}{ foams $(n=5)$} & \multicolumn{4}{|c|}{ ropes $(n=3)$} & \multicolumn{4}{|c|}{ painted surfaces $(n=6)$} \\
\hline & $n>\mathrm{LOD}$ & median & $\min$ & $\max$ & $n>$ LOD & edian & $\min$ & $\max$ & $n>\mathrm{LOD}$ & median & $\min$ & $\max$ & $n>$ LOD & median & $\min$ & $\max$ \\
\hline As & 4 & 14.2 & 6.0 & 24.6 & 2 & 12.0 & 10.5 & 13.5 & 3 & 13.0 & 10.6 & 21.1 & 4 & 5.2 & 0.6 & 19.9 \\
\hline $\mathrm{Ba}$ & 2 & 10.4 & 3.1 & 17.7 & 0 & & & & 0 & & & & 2 & 4.8 & 1.3 & 8.3 \\
\hline $\mathrm{Bi}$ & 0 & & & & 0 & & & & 0 & & & & 1 & 24.6 & 24.6 & 24.6 \\
\hline $\mathrm{Br}$ & 0 & & & & 5 & 4.5 & 1.5 & 12.0 & 1 & 1.3 & 1.3 & 1.3 & 3 & 4.8 & 1.1 & 10.6 \\
\hline $\mathrm{Cd}$ & 3 & 1.6 & 0.6 & 7.9 & 0 & & & & 0 & & & & 0 & & & \\
\hline $\mathrm{Cl}$ & 5 & 5.9 & 3.1 & 11.5 & 5 & 1.6 & 0.6 & 4.3 & 3 & 6.4 & 2.9 & 7.5 & 6 & 2.7 & 0.1 & 17.0 \\
\hline $\mathrm{Cr}$ & 6 & 3.9 & 1.1 & 13.8 & 4 & 4.7 & 4.0 & 7.9 & 3 & 2.3 & 1.5 & 2.5 & 0 & & & \\
\hline $\mathrm{Cu}$ & 1 & 16.5 & 16.5 & 16.5 & 4 & 13.8 & 9 & 21.8 & 1 & 7.5 & 7.5 & 7.5 & 0 & & & \\
\hline $\mathrm{Fe}$ & 5 & 3.1 & 1.2 & 8.8 & 5 & 3.6 & 0.8 & 11.1 & 3 & 4.0 & 0.8 & 18.2 & 6 & 3.7 & 0.1 & 4.7 \\
\hline $\mathrm{Hg}$ & 0 & & & & 0 & & & & 0 & & & & 0 & & & \\
\hline $\mathrm{Ni}$ & 2 & 17.6 & 15.9 & 19.4 & 0 & & & & 0 & & & & 0 & & & \\
\hline $\mathrm{Pb}$ & 4 & 1.7 & 0.9 & 4.3 & 2 & 4.0 & 3.1 & 4.8 & 3 & 2.6 & 1.3 & 3.6 & 5 & 2.0 & 0.4 & 16.5 \\
\hline $\mathrm{Sb}$ & 3 & 9.8 & 2.3 & 18.4 & 0 & & & & 2 & 21.7 & 17.8 & 25.5 & 0 & & & \\
\hline $\mathrm{Se}$ & 1 & 2.9 & 2.9 & 2.9 & 0 & & & & 0 & & & & 0 & & & \\
\hline Sn & 0 & & & & 2 & 12.6 & 8.7 & 16.5 & 0 & & & & 0 & & & \\
\hline $\mathrm{Ti}$ & 6 & 2.6 & 0.6 & 10.8 & 5 & 8.7 & 1.5 & 21.2 & 2 & 2.5 & 2.5 & 2.5 & 6 & 0.9 & 0.5 & 6.4 \\
\hline $\mathrm{Zn}$ & 6 & 4.7 & 2.0 & 14.2 & 4 & 7.7 & 5.6 & 10.0 & 1 & 15.7 & 15.7 & 15.7 & 6 & 3.1 & 1.1 & 7.7 \\
\hline
\end{tabular}


Table 4: Number of cases detected and median, minimum and maximum concentrations $\left(\mu \mathrm{g} \mathrm{g}^{-1}\right)$ for each element and in the four categories of litter for a total counting time of 120 seconds and with appropriate thickness correction applied. Original res version in Excel file.

\begin{tabular}{|c|c|c|c|c|c|c|c|c|c|c|c|c|c|c|c|c|}
\hline & \multicolumn{4}{|c|}{ plastics $(n=149)$} & \multicolumn{4}{|c|}{ foams $(n=149)$} & \multicolumn{4}{|c|}{ ropes $(n=68)$} & \multicolumn{4}{|c|}{ painted surfaces $(n=10)$} \\
\hline & $n>$ LOD & median & $\min$ & $\max$ & $n>$ LOD & median & $\min$ & $\max$ & $n>\mathrm{LOD}$ & median & $\min$ & $\max$ & $n>$ LOD & median & $\min$ & $\max$ \\
\hline As & 1 & 6.2 & 6.2 & 6.2 & 9 & 56 & 25 & 210 & 2 & 27 & 17 & 36 & 0 & & & \\
\hline $\mathrm{Ba}$ & 17 & 1710 & 329 & 10,700 & 9 & 1090 & 298 & 20,400 & 0 & & & & 2 & 4070 & 4030 & 4100 \\
\hline $\mathrm{Bi}$ & 4 & 88 & 20 & 198 & 0 & & & & 0 & & & & 1 & 79 & 79 & 79 \\
\hline $\mathrm{Br}$ & 23 & 32 & 4.8 & 1180 & 112 & 218 & 12 & 17,600 & 32 & 20 & 6.6 & 91 & 7 & 37 & 5.6 & 61 \\
\hline $\mathrm{Cd}$ & 5 & 1940 & 117 & 4640 & 0 & & & & 0 & & & & 0 & & & \\
\hline $\mathrm{Cl}$ & 127 & 594 & 4.3 & 408,000 & 143 & 60,700 & 744 & 572,000 & 63 & 958 & 189 & 20,400 & 10 & 39 & 9.6 & 614 \\
\hline $\mathrm{Cr}$ & 77 & 34 & 21 & 717 & 108 & 210 & 23 & 940 & 32 & 63 & 25 & 909 & 0 & & & \\
\hline $\mathrm{Cu}$ & 35 & 60 & 22 & 718 & 29 & 203 & 71 & 1910 & 47 & 76 & 27 & 808 & 2 & 14.0 & 10 & 18 \\
\hline $\mathrm{Fe}$ & 121 & 136 & 20 & 28,700 & 148 & 7570 & 279 & 120,000 & 67 & 1160 & 88 & 29,200 & 10 & 90 & 11 & 400 \\
\hline $\mathrm{Hg}$ & 1 & 272 & 272 & 272 & 0 & & & & 0 & & & & 2 & 7.3 & 4.0 & 11 \\
\hline $\mathrm{Ni}$ & 3 & 29 & 19 & 74 & 2 & 220 & 220 & 220 & 2 & 386 & 37 & 735 & 0 & & & \\
\hline $\mathrm{Pb}$ & 19 & 58 & 6.3 & 13,200 & 29 & 119 & 6.7 & 17,000 & 28 & 199 & 13 & 3770 & 6 & 175 & 3.6 & 10,700 \\
\hline $\mathrm{Sb}$ & 5 & 228 & 154 & 6260 & 4 & 259 & 67 & 5819 & 0 & & & & 0 & & & \\
\hline $\mathrm{Se}$ & 2 & 457 & 351 & 563 & 0 & & & & 0 & & & & 0 & & & \\
\hline Sn & 3 & 486 & 35 & 2090 & 22 & 209 & 53 & 2280 & 1 & 83 & 83 & 83 & 0 & & & \\
\hline $\mathrm{Ti}$ & 136 & 387 & 5.6 & 63,900 & 143 & 867 & 18 & 30,400 & 67 & 386 & 16.8 & 3140 & 10 & 2920 & 3.5 & 454 \\
\hline $\mathrm{Zn}$ & 73 & 39 & 13 & 26,700 & 89 & 229 & 25 & 25,300 & 28 & 30 & 15 & 535 & 7 & 92 & 6.0 & 1570 \\
\hline
\end{tabular}


Table 5: Parameters defining the relationships between FP-XRF and ICP-OES measurements of various marine litter samples. The slope, $m$, correlation coefficient (significant at $p<0.01$ ), $r$, and number of data points, $n$, are shown for each element and, for $\mathrm{Cu}$ and $\mathrm{Zn}($ and in parentheses), after exclusion of two foam samples. Original res version in Excel file.

\begin{tabular}{lccc}
\hline element & $m$ & $r$ & $n$ \\
\hline $\mathrm{Ba}$ & $1.48^{*}$ & $\mathrm{~ns}$ & 4 \\
$\mathrm{Cd}$ & 1.05 & 0.969 & 4 \\
$\mathrm{Cr}$ & 1.33 & 0.550 & 12 \\
$\mathrm{Cu}$ & $8.02(1.72)$ & $0.849(0.871)$ & $7(5)$ \\
$\mathrm{Fe}$ & 0.75 & 0.948 & 15 \\
$\mathrm{~Pb}$ & 1.39 & 0.795 & 11 \\
$\mathrm{Sb}$ & 1.34 & 0.999 & 3 \\
$\mathrm{Se}$ & 1.18 & 0.999 & 2 \\
$\mathrm{Sn}$ & 4.03 & 0.997 & 3 \\
$\mathrm{Ti}$ & 1.17 & 0.984 & 18 \\
$\mathrm{Zn}$ & $2.54(1.59)$ & $0.641(0.999)$ & $12(10)$ \\
\hline
\end{tabular}

*Since the relationship for Ba was not significant, the slope is represented as the average ratio defining the concentrations derived from the two approaches. 
Figure 1: Elemental concentrations in the reference polyethylene disc, PN-180-554, as a function of thickness correction applied and for a total counting time of 120 seconds. Note that concentrations are normalised to values shown in the legend (in $\mu \mathrm{g} \mathrm{g}^{-1}$ and derived without thickness correction). High res version in Excel file.

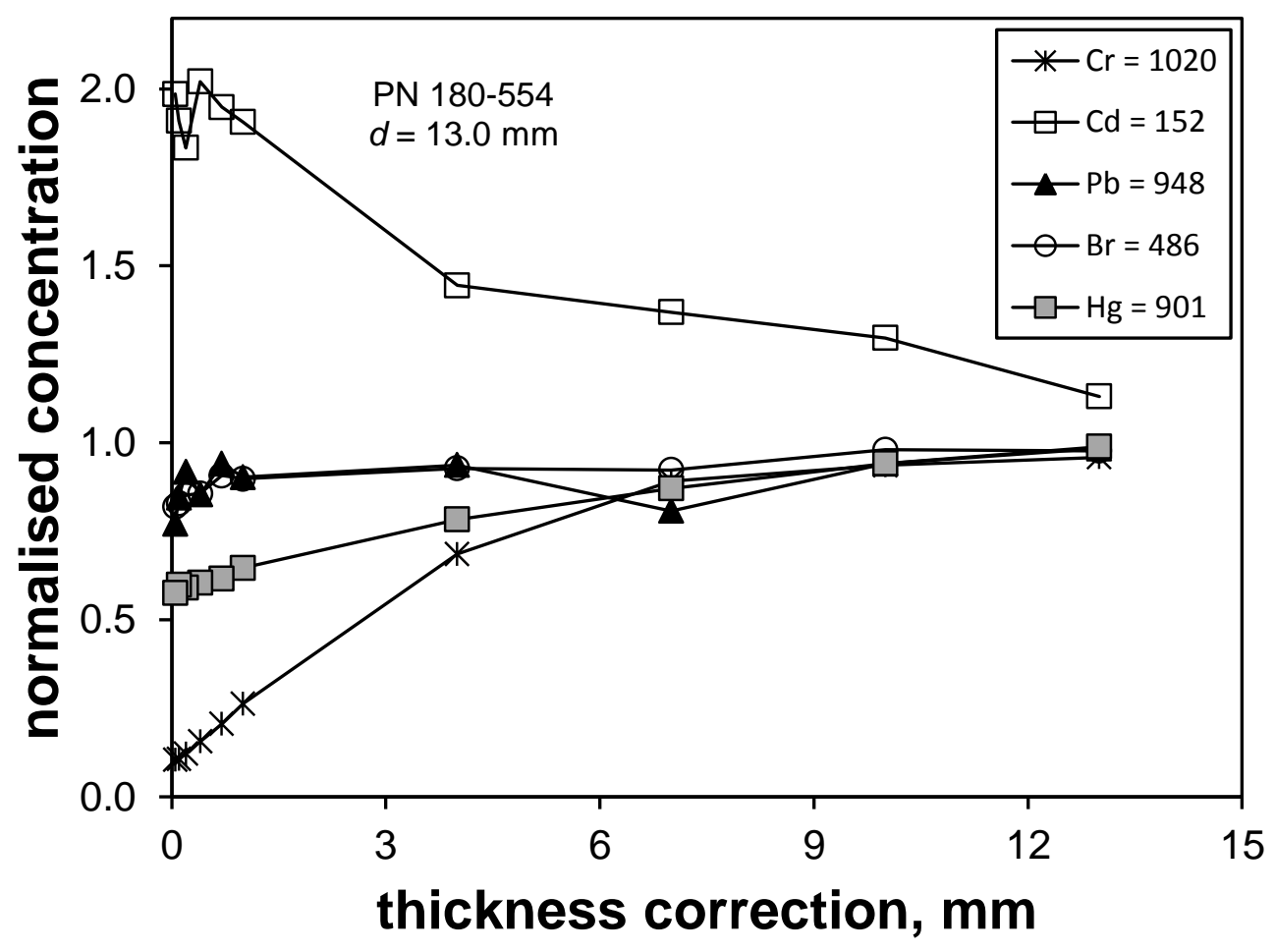


Figure 2: Elemental concentrations in various plastic (polyethylene and polypropylene) litter samples as a function of thickness correction applied and for a total counting time of 120 seconds. Note that concentrations are normalised to values shown in the legend (in $\mu \mathrm{g} \mathrm{g}^{-1}$ and derived with appropriate thickness correction). High res version in Excel file. 

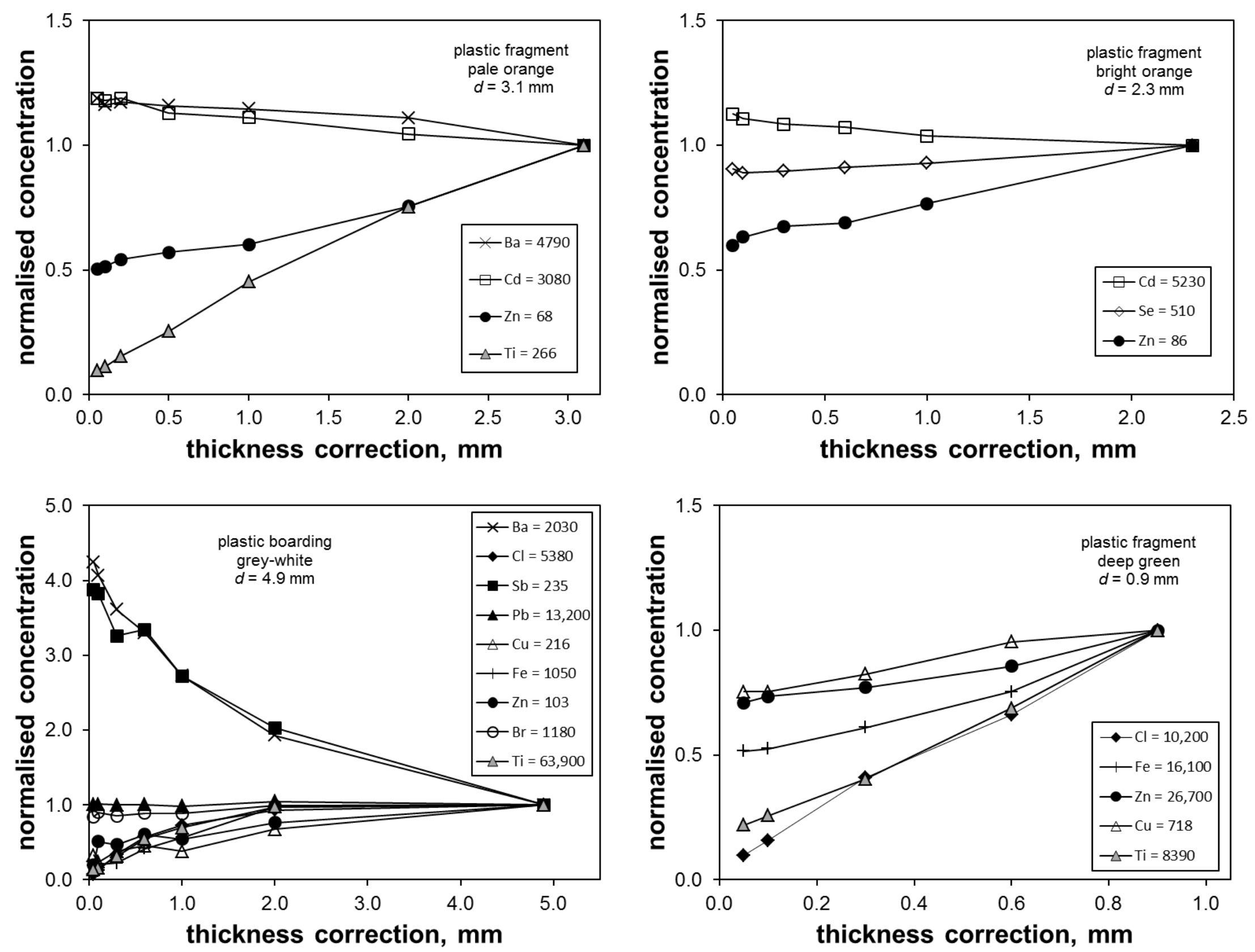
Figure 3: Elemental concentrations in various foams (including a fragment of cork) and ropes as a function of thickness correction applied and for a total counting time of 120 seconds. Note that concentrations are normalised to values shown in the legend (in $\mu \mathrm{g} \mathrm{g}^{-1}$ and derived with appropriate thickness correction). $\mathrm{EPS}=$ expanded polystyrene; $\mathrm{EPU}=$ expanded polyurethane. High res version in Excel file.
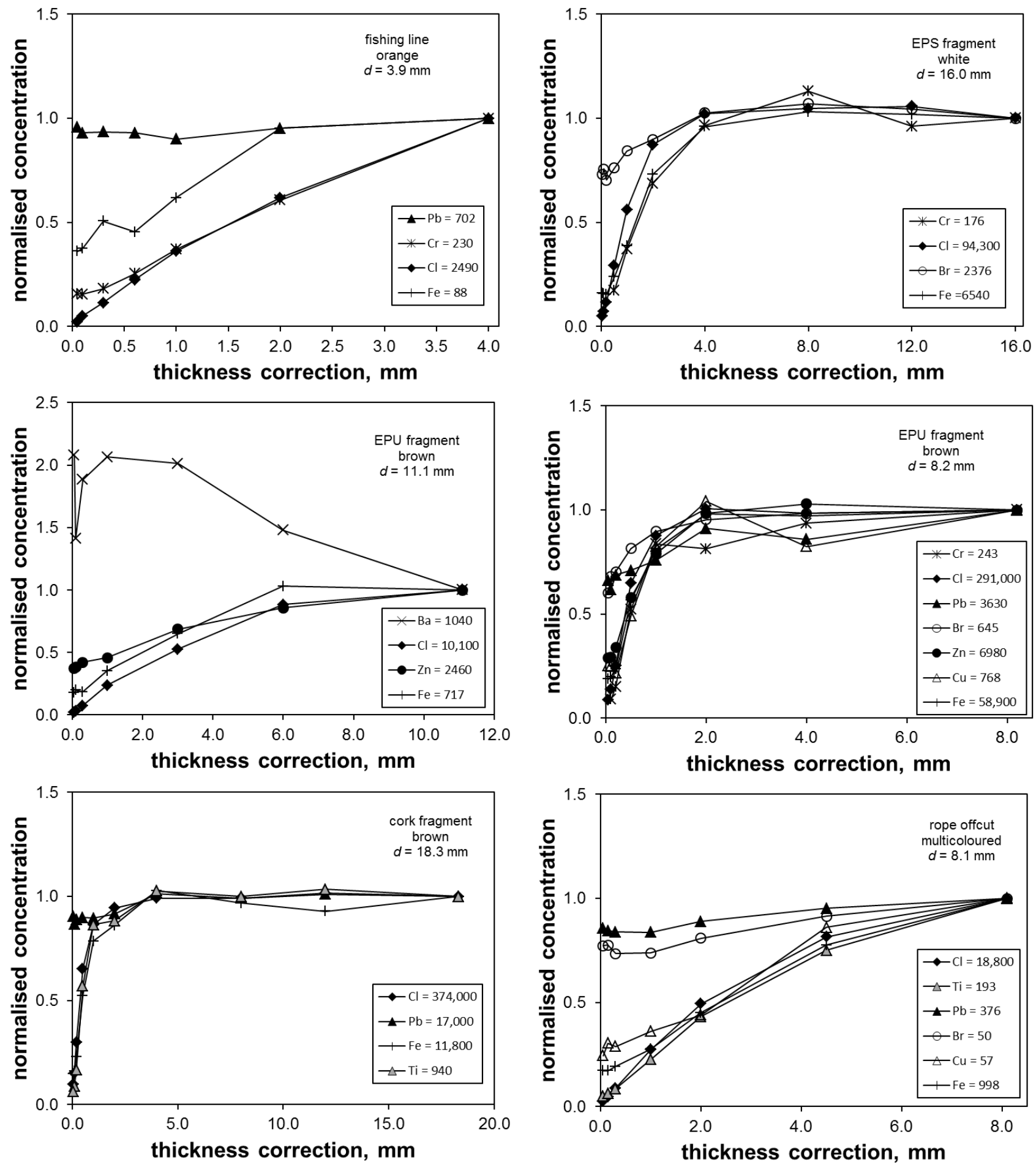
Figure 4: Spatial distribution of various elements (in $\mu \mathrm{g} \mathrm{g}^{-1}$ ) measured at the surface of a fragment of painted acrylic-fibreglass with resinous backing. (for colour reproduction on the web)

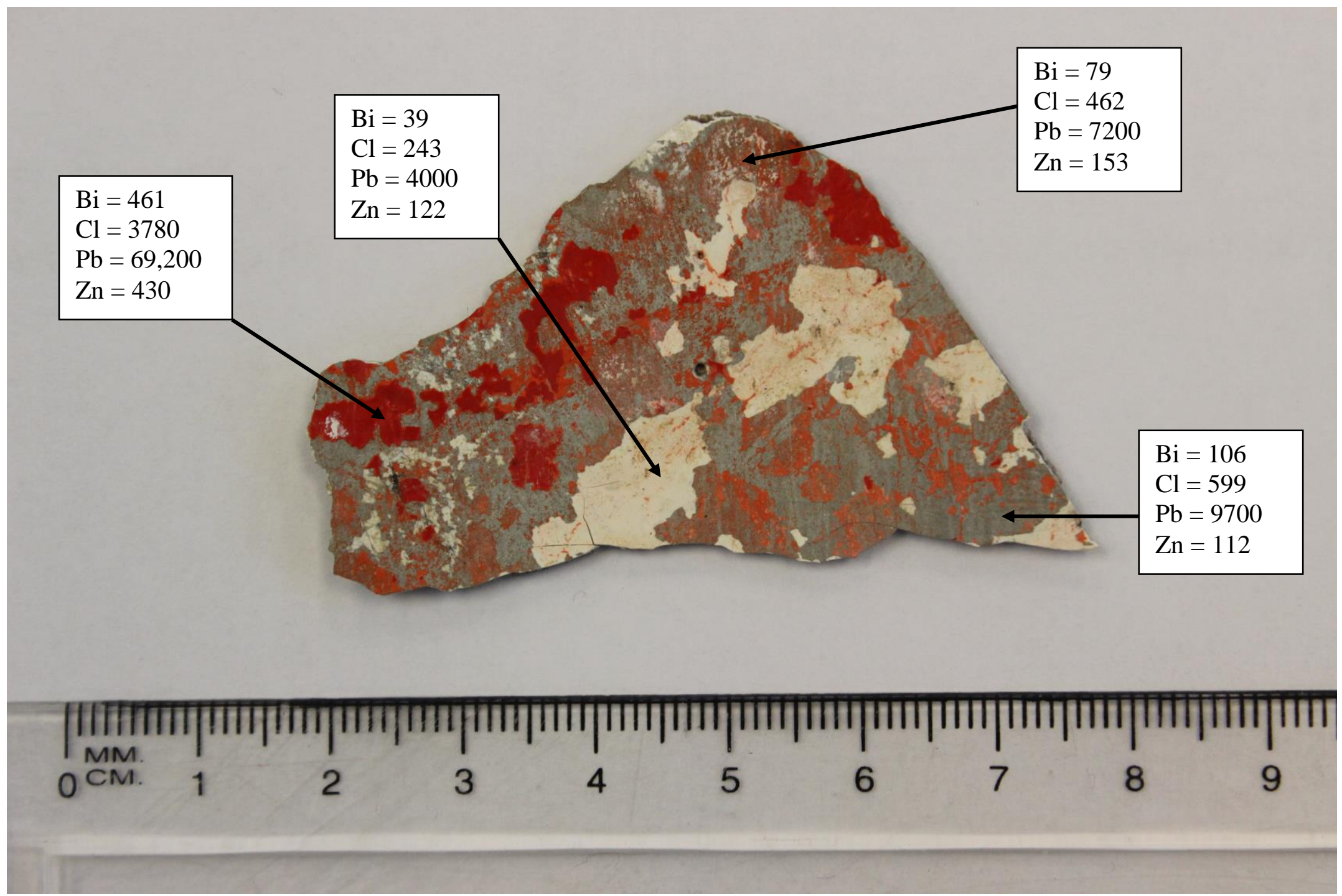


Figure 5: Spatial and depth distribution of various elements (in $\mu \mathrm{g} \mathrm{g}^{-1}$ ) in a fragment of cork that had been sliced with a stainless steel scalpel. (for colour reproduction on the web)

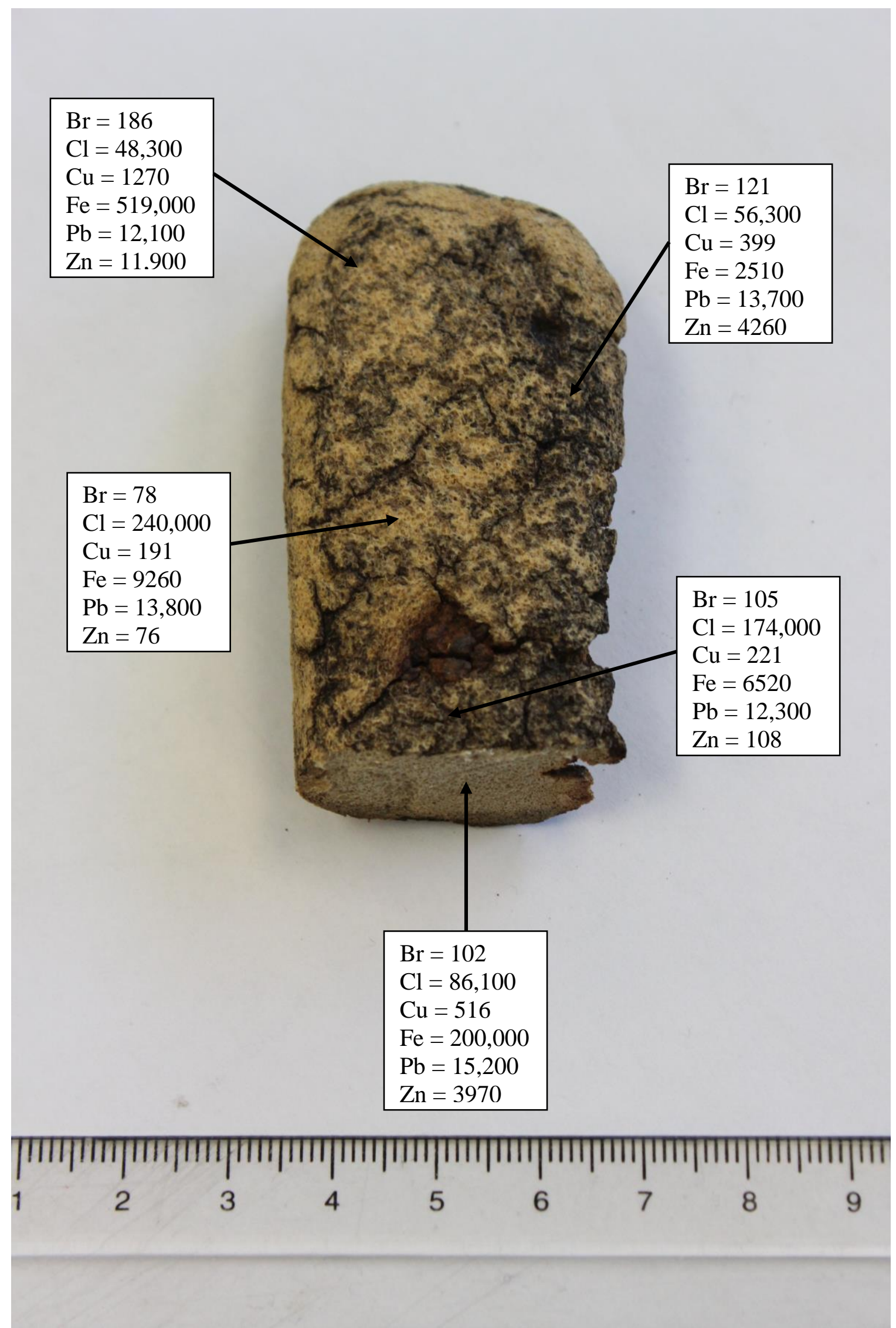


Figure 6: A comparison of the concentrations of elements in various samples of marine litter returned by the FP-XRF and by ICP-OES following acid digestion. Annotated are lines defining unit slope and ratios of [XRF] to [ICP] of 10:1 and 1:10. High res version in Excel file.

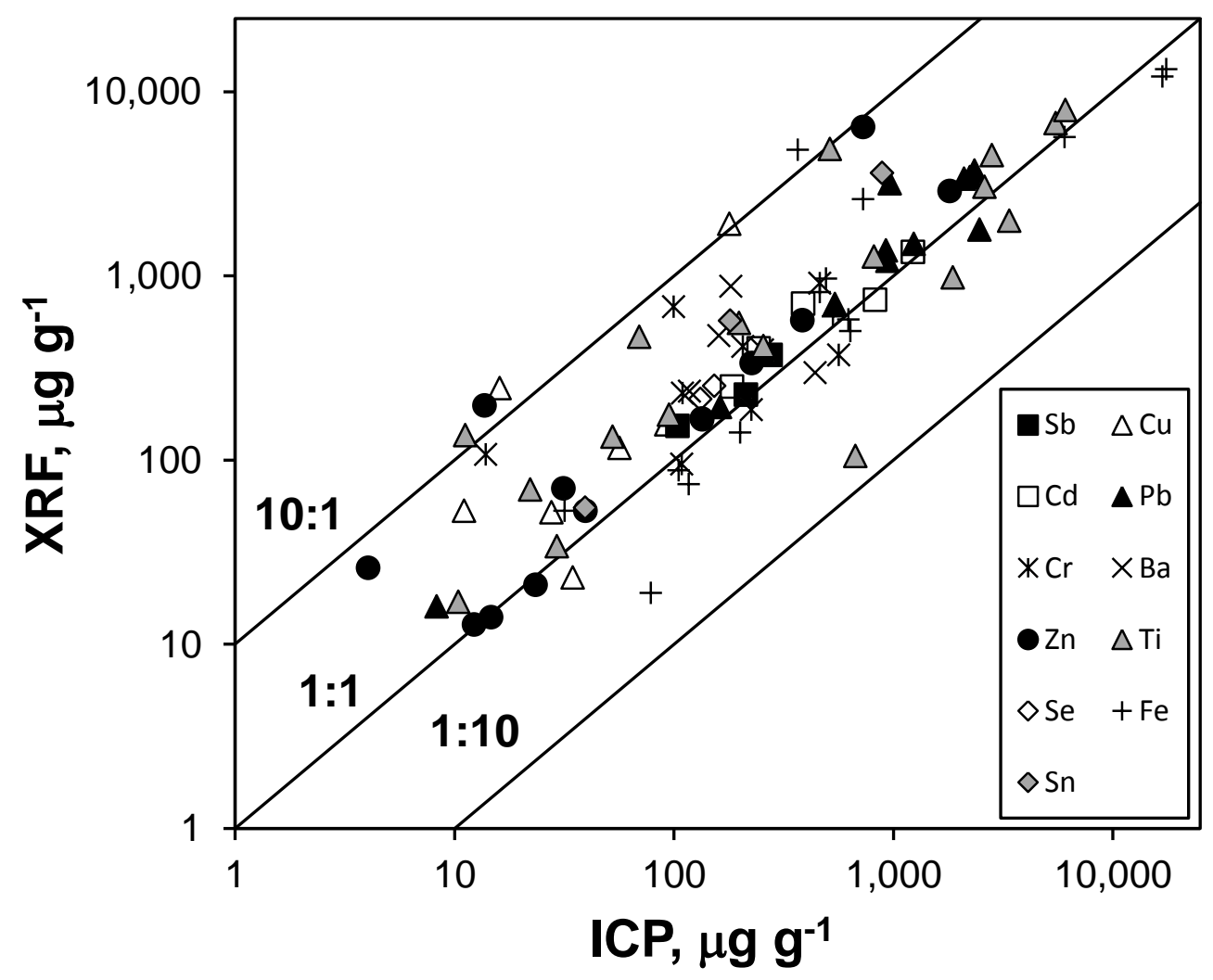

